PREPARED FOR THE U.S. DEPARTMENT OF ENERGY, UNDER CONTRACT DE-AC02-76CH03073

PPPL-3925

PPPL-3925

UC-70

\title{
Anomalous Skin Effect for Anisotropic Electron \\ Velocity Distribution Function
}

by

Igor Kaganovich, Edward Startsev, and Gennady Shvets

February 2004

M

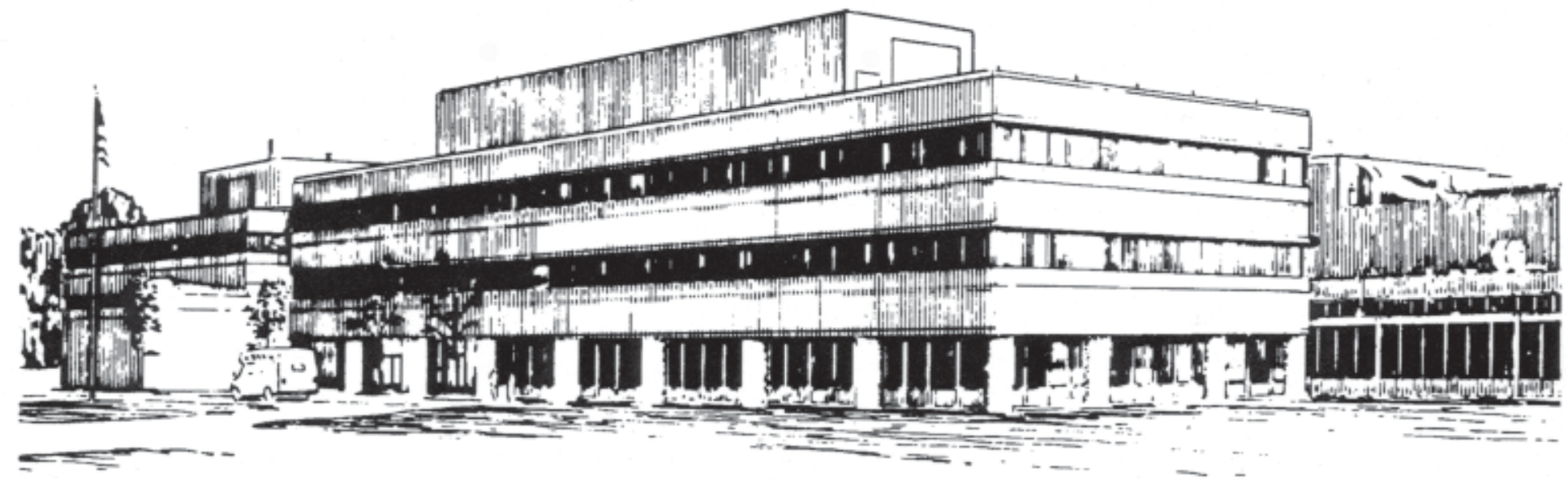

PRINCETON PLASMA PHYSICS LABORATORY PRINCETON UNIVERSITY, PRINCETON, NEW JERSEY 


\section{PPPL Reports Disclaimer}

This report was prepared as an account of work sponsored by an agency of the United States Government. Neither the United States Government nor any agency thereof, nor any of their employees, makes any warranty, express or implied, or assumes any legal liability or responsibility for the accuracy, completeness, or usefulness of any information, apparatus, product, or process disclosed, or represents that its use would not infringe privately owned rights. Reference herein to any specific commercial product, process, or service by trade name, trademark, manufacturer, or otherwise, does not necessarily constitute or imply its endorsement, recommendation, or favoring by the United States Government or any agency thereof. The views and opinions of authors expressed herein do not necessarily state or reflect those of the United States Government or any agency thereof.

\section{Availability}

This report is posted on the U.S. Department of Energy's Princeton Plasma Physics Laboratory Publications and Reports web site in Fiscal Year 2004. The home page for PPPL Reports and Publications is: http://www.pppl.gov/pub_report/

DOE and DOE Contractors can obtain copies of this report from:

U.S. Department of Energy

Office of Scientific and Technical Information

DOE Technical Information Services (DTIS)

P.O. Box 62

Oak Ridge, TN 37831

Telephone: (865) 576-8401

Fax: (865) 576-5728

Email: reports@adonis.osti.gov

This report is available to the general public from:

National Technical Information Service

U.S. Department of Commerce

5285 Port Royal Road

Springfield, VA 22161

Telephone: $1-800-553-6847$ or

(703) $605-6000$

Fax: (703) 321-8547

Internet: http://www.ntis.gov/ordering.htm 


\title{
Anomalous skin effect for anisotropic electron velocity distribution function
}

\author{
Igor Kaganovich, Edward Startsev \\ Princeton Plasma Physics Laboratory, \\ Princeton University, Princeton, New Jersey 08543
}

Gennady Shvets

University of Texas at Austin, Institute for Fusion Studies, Austin, Texas 78712

\begin{abstract}
The anomalous skin effect in a plasma with a highly anisotropic electron velocity distribution function $(\mathrm{EVDF})$ is very different from skin effect in a plasma with the isotropic EVDF. An analytical solution was derived for the electric field penetrated into plasma with the EVDF described as a Maxwellian with two temperatures $T_{x}>>T_{z}$, where $x$ is the direction along the plasma boundary and $z$ is the direction perpendicular to the plasma boundary. The skin layer was found to consist of two distinctive regions of width of order $v_{T x} / \omega$ and $v_{T z} / \omega$, where $v_{T x, z}=\sqrt{T_{x, z} / m}$ is the thermal electron velocity and $\omega$ is the incident wave frequency.
\end{abstract}


In a recent Letter [1], it was shown that a highly anisotropic electron velocity distribution function (EVDF) yields a large skin-layer depth compared with the isotropic EVDF. The EVDF was described as a Maxwellian with two temperatures $T_{x}>>T_{z}$, where $x$ is the direction along plasma boundary and $z$ is the direction perpendicular plasma boundary. The electromagnetic wave is assumed to propagate also along $z$-axis in vacuum. The skin layer was found to be much longer than the skin layer in a plasma with isotropic EVDF. The authors of Ref. [1] showed that under conditions

$$
T_{x}>>T_{z} ; \frac{c}{\omega_{p}}<<\frac{v_{T x}}{\omega} ; \omega_{p}>>\omega
$$

where $\omega$ is the incident wave frequency, $\omega_{p}=\sqrt{4 \pi e^{2} n / m}$ is the plasma frequency, $n$ is the electron density, $v_{T x}=\sqrt{T_{x} / m}$, the electric field profile is exponential $E(z) \sim \exp \left(-z / l_{s}\right)$ where

$$
l_{s}=\frac{v_{T x}}{\omega} .
$$

In their analysis authors of Ref.[1] assumed from the outset that the skin depth is much longer than $v_{T z} / \omega$, where $v_{T z}=\sqrt{T_{z} / m}, T_{z}$ is the electron temperature along $z$-axis perpendicular to the plasma boundary. We show that the skin layer actually consists of two distinctive regions of widths of order $v_{T x} / \omega$ and $v_{T z} / \omega$. The latter short region was missed in Ref. [1].

In contrast to Ref. [1], we solve Maxwell's equation

$$
\frac{d^{2}}{d z^{2}} E(z)+\frac{\omega^{2}}{c^{2}} E(z)=-\frac{4 \pi i \omega}{c^{2}} j_{x}
$$

for $x$ - component of electric field without making any assumptions. For semi-infinite geometry, the electric field can be calculated making use of the Fourier transform in the infinite plane by continuing the electric field symmetrically around plasma boundary $[E(-z)=E(z)]$. Following Ref. [2], the Fourier transform of the electric field is given by

$$
E(k)=-\frac{2 i \omega}{c} B(0) \frac{1}{k^{2}-\varepsilon_{t}(\omega, k) \omega^{2} / c^{2}},
$$

where $B(0)$ is the magnetic field at plasma boundary and $\varepsilon_{t}(\omega, k)$ is the transverse plasma dielectric constant [2]

$$
\varepsilon_{t}(\omega, k)=1-\frac{4 \pi i}{\omega E(k)} e \int v_{x} \delta f d \mathbf{v}
$$


where $\delta f$ is the perturbation of electron velocity distribution function due to a planar $x$-polarized electromagnetic wave with frequency $\omega$ and wavenumber $\vec{k}=k \vec{e}_{z}$. To determine $\delta f$ and consequently $\varepsilon_{t}$ we perform the Fourier transform of the Vlasov equation $[1]:$

$$
\delta f(k)=-\frac{e}{i m}\left[\frac{E(k)-v_{z} B(k) / c}{\omega-v_{z} k} \frac{\partial f_{0}}{\partial v_{x}}+\frac{v_{x} B(k) / c}{\omega-v_{z} k} \frac{\partial f_{0}}{\partial v_{z}}\right] .
$$

Because in the planar electromagnetic wave $B(k)=c k E(k) / \omega$, Eq.(5) simplifies to

$$
\varepsilon_{t}(\omega, k)=1+\frac{4 \pi e^{2}}{m \omega^{2}} \int d \mathbf{v}\left[v_{x} \frac{\partial f_{0}}{\partial v_{x}}+\frac{v_{x}^{2} k}{\left(\omega-v_{z} k\right)} \frac{\partial f_{0}}{\partial v_{z}}\right]
$$

Substituting $f_{0}$ as a Maxwellian with two different temperatures $T_{x}$ and $T_{z}$ into Eq.(7) and making use of an algebraic identity

$$
\frac{v_{x}^{2} k}{\left(\omega-v_{z} k\right)} \frac{\partial f_{0}}{\partial v_{z}}=\frac{m v_{x}^{2}}{T_{z}} f_{0}\left(1+\frac{\omega / v_{T z} k}{v_{z} / v_{T z}-\omega / v_{T z} k}\right)
$$

gives

$$
\varepsilon_{t}(\omega, k)=1-\frac{\omega_{p}^{2}}{\omega^{2}}\left\{1-\frac{T_{x}}{T_{z}}\left[1+\frac{\omega}{\sqrt{2} v_{T z} k} Z\left(\frac{\omega}{\sqrt{2} v_{T z} k}\right)\right]\right\} .
$$

where $Z(\zeta)$ is the plasma dielectic function [2].

The spatial profile of the electric field $E(z)$ is given by the inverse Fourier transform of Eq.(4)

$$
E(z)=-\frac{i \omega}{\pi c} B(0) \int_{-\infty}^{\infty} \frac{e^{i k z}}{k^{2}-\varepsilon_{t}(\omega,|k|) \omega^{2} / c^{2}} d k .
$$

The $|k|$ denotes the fact that $E(z)$ is continued symmetrically to the semi-plane $z<0$ and $E(z)=E(-z)$, which is satisfied by setting $E(k)=E(-k)$ [2]. Note that despite $E(z)=E(-z)$, the derivative of $E(z)$ is not continues at $z=0$.

The contour of integration in Eq.(10) can be shifted into complex $k$-plane. Because $|k|=\sqrt{k^{2}}$, the contour of integration has to enclose the branch point $k=0$ with the cut along the imaginary $k$ axis [3]. As a result Eq.(10) can be represented as a sum of contributions from poles and an integral along the imaginary axis of the complex $k$-plane

$$
\begin{aligned}
E(z)= & -\frac{i \omega}{\pi c} B(0) \times \\
& \left\{\sum_{n} e^{i k_{p n} z} 2 \pi i \operatorname{Rez}\left(\frac{1}{k_{p n}^{2}-\varepsilon_{t}\left(\omega, k_{p n}\right) \omega^{2} / c^{2}}\right)\right. \\
& \left.+\int_{0}^{\infty} \frac{\operatorname{Im} \varepsilon_{t}(\omega, i s) \omega^{2} / c^{2} e^{-s z}}{\left[s^{2}+\operatorname{Re} \varepsilon_{t}(\omega, i s) \omega^{2} / c^{2}\right]^{2}+\left[\operatorname{Im} \varepsilon_{t}(\omega, i s) \omega^{2} / c^{2}\right]^{2}} d s\right\} .
\end{aligned}
$$


Here, $k_{p n}$ are the poles of denominator in Eq.(10) in the complex k-plane given by

$$
k_{p n}^{2}-\omega^{2} / c^{2}+\omega_{p}^{2} / c^{2}\left\{1-\frac{T_{x}}{T_{z}}\left[1+\frac{\omega}{\sqrt{2} v_{T z} k_{p n}} Z\left(\frac{\omega}{\sqrt{2} v_{T z} k_{p n}}\right)\right]\right\}=0 .
$$

In the limit of small $k, \omega / \sqrt{2}|k| v_{T z} \equiv \zeta>>1$ and $1+\zeta Z(\zeta) \rightarrow-1 / 2 \zeta^{2}$,

$$
\varepsilon_{t}(\omega, k)=1-\frac{\omega_{p}^{2}}{\omega^{2}}\left(1+\frac{v_{T x}^{2} k^{2}}{\omega^{2}}\right)
$$

and the pole is

$$
k_{p 1}^{2}=-\frac{\left(\omega_{p}^{2}-\omega^{2}\right)}{c^{2}+\omega_{p}^{2} v_{T x}^{2} / \omega^{2}} .
$$

Under the conditions Eq.(1), Eq.(15) simplifies to become

$$
k_{p 1}=i \frac{\omega}{v_{T x}} .
$$

Calculations of the residual in Eq.(11) gives the electric field profile from this pole

$$
E_{p 1}(z)=-\frac{i \omega^{2} c}{\omega_{p}^{2} v_{T x}} B(0) e^{i k_{p 1} z}
$$

This corresponds to the exponential decay of the electric field with the scale $l_{s}=v_{T x} / \omega$ described in Ref. [1].

However, there is another pole $k_{p 2}>>k_{p 1}$. In the limit of large $k, \omega / \sqrt{2}|k| v_{T z} \equiv \zeta<<1$ $\zeta Z(\zeta)<<1$ and under the conditions in Eq.(1), Eq.(13) yields

$$
\operatorname{Re}_{p 2}=\frac{\omega_{p}}{c} \sqrt{T_{x} / T_{z}}
$$

Note that according to Eq.(1)

$$
\frac{\omega}{k_{p 2} v_{T z}}=\frac{\omega c}{\omega_{p} v_{T x}}<<1
$$

Imaginary part of $k_{p 2}$ can be determined taking into account imaginary part of $Z(0)=i \sqrt{\pi}$, which gives

$$
\operatorname{Im} k_{p 2}=\frac{\sqrt{\pi} \omega}{2 \sqrt{2} v_{T z}} .
$$

The pole $k_{p 2}$ gives rise to the rapidly oscillating field in the plasma

$$
E_{p 2}(z)=\frac{\omega}{c k_{p 2}} B(0) e^{i k_{p 2} z}
$$

Under the conditions Eq.(1), the contribution of the branch point [last integral in Eq.(11)] is small. Indeed, the width of the integral is determined by the dispersion function and it is 
equal to $\omega / v_{T z}$ while the amplitude of the function under the integral is of order $c^{2} T_{z} / \omega_{p}^{2} T_{x}$. This gives for the contribution from the branch point $E_{b}(z)$ an estimate

$$
E_{b}(z) \sim B(0) \frac{\omega^{2} c \sqrt{T_{z} / T_{x}}}{\pi v_{T x} \omega_{p}^{2}},
$$

which is $2 \pi \sqrt{T_{x} / T_{z}}$ times smaller than $E_{p 1}(z)$ in Eq.(17). Note that it is in contrast to the classical anomalous skin effect, where the contribution of the branch point is comparable to the pole contribution [3].

Exact numerical integration of inverse Fourier transform of Eq.(10) confirms the importance of the oscillating solution as shown in Fig.1. Therefore the prediction of Ref. [1] of monotonically decaying electric field is inaccurate.

Finally, the profile of the electric field is a sum of the two complex exponents given by Eq.(17) and Eq.(21)

$$
E(z)=E_{p 1} \exp \left(-i k_{p 1} z\right)+E_{p 2} \exp \left(-i k_{p 2} z\right)
$$

with $k_{p 1}$ given by Eq.(16) and $k_{p 2}$ given by Eqs.(18) and (20). The first pole in Eq.(23) produces a slowly decaying electric field, while the second pole produces a faster decaying electric field $\left(R e k_{p 2}>>R e k_{p 1}\right)$. Note that, in contrast to anomalous skin effect in plasma with isotropic EVDF, the skin layer in a plasma with anisotropic EVDF consists of two distinctive layers with very different lengthes. The amplitude of short layer $E_{p 2}$ is larger in most cases than the amplitude of long layer $E_{p 1}$. It follows from Eq.(17) and Eq.(21) that

$$
\frac{\left|E_{p 2}\right|}{\left|E_{p 1}\right|} \sim \frac{\omega_{p} v_{T x}}{\omega c} \frac{1}{\sqrt{T_{x} / T_{z}}},
$$

and under conditions in Eq.(1) amplitude of the electric field $E_{p 2}$ is large compared with $E_{p 1}$ for modest anisotropy $\left(\sqrt{T_{x} / T_{z}} \sim 1\right)$, whereas amplitudes are comparable for very large anisotropy $\left(\sqrt{T_{x} / T_{z}}>>1\right)$, as can be seen in Fig.1.

The surface impedance - the ratio of the electric and magnetic fields at the boundary characterizes the absorption coefficient and the phase of reflected wave $[2,4]$. Substituting Eqs.(17) and (21) [together with Eqs.(18) and (20)] gives

$$
\zeta=\frac{E(0)}{B(0)}=-\frac{i \omega^{2} c}{\omega_{p}^{2} v_{T x}}+\frac{\omega}{\omega_{p} \sqrt{T_{x} / T_{z}}+i \frac{c \sqrt{\pi} \omega}{2 \sqrt{2} v_{T z}}} .
$$

The energy dissipation in the plasma and, correspondingly the absorption coefficient are determined by the real part of the surface impedance. Under the conditions (1), it follows 
from Eq.(25) that the real part of the surface impedance can be expressed as

$$
R e(\zeta)=\frac{\omega}{\omega_{p} \sqrt{T_{x} / T_{z}}} .
$$

Therefore, the absorption coefficient in semi-infinite plasma is entirely governed by the short scale region of width of order $v_{T z} / \omega$. Equation (26) recovers the result previously obtained in Ref.[5].

Generally speaking, the anisotropic EVDF is the subject of the Weibel instability [6]. The growth rate can be obtained analyzing the poles of Eq.(13) with real $k$, but complex $\omega$. The maximum growth rate is given by $\gamma=\omega_{p} v_{T x} / c[6]$. Instability develops faster than one laser oscillation. Indeed, $\gamma / \omega=\omega_{p} v_{T x} / c \omega=\omega_{p} / \omega\left(v_{T x} / c\right)$. The last parameter is large according to the assumption in Eq.(1) and, therefore, the instability has time to develop. However, particle-in-cell simulations in Ref. [7] shows that Weibel instability may saturate on relatively low levels where the EVDF remains very anisotropic.

In summary, we have discovered that the electric field structure in the skin layer is far from a monotonic exponentially decaying profile predicted in [1]. In fact, the skin layer contains multiple oscillations of the electric field. The non-monotonic nature of the electric field decay accounts for the finite dissipation missed in Ref. [1]. The anisotropic EVDF is the subject of the Weibel instability, which develops quickly during the penetration of the electric field into the plasma. However, the Weibel instability may saturate on relatively low levels where the EVDF remains very anisotropic. The exact estimates of the saturation level are difficult analytically and, therefore, self consistent particle-in-cell simulations are necessary for further investigation of the subject.

This research was supported by the U.S. Department of Energy and (for G.S.) by the DOE Office of Fusion Energy Sciences Junior Faculty Award. 
[1] G. Ferrante, M. Zarcone and S.A. Urypin, Phys. Rev. Lett. 91, 085005 (2003).

[2] E.M. Lifshitz and L.P. Pitaevskii, "Physical Kinetics", ( Pergamon Press, Oxford 1981), p.368.

[3] Yu. M. Aliev, I. D. Kaganovich and H. Schluter, Phys. Plasmas, 4, 2413 (1997); and in more details Yu. M. Aliev, I. D. Kaganovich and H. Schluter, "Collisionless electron heating in RF gas discharges. I. Quasilinear theory" in U.Korsthagen and L. Tsendin (Eds.), Electron kinetics and Applications of glow discharges, NATO ASI Series B, Physics 367, Plenum Press, (New York and London) (1998).

[4] A.A. Andreev, K. Yu. Platonov and J.C. Gauthier, Phys. Rev. E 58, 2424 (1998).

[5] G. Ferrante, M. Zarcone and S.A. Urypin, Eur. Phys. D. 19, 349 (2002).

[6] E.S. Weibel, Phys. Rev. Lett. 2, 83 (1959).

[7] M. Honda, J. Meyer-ter-Vehn, A. Pukhov, Phys. Plasmas 7, 1302 (2000).

\section{FIGURE CAPTION}

The electric field in plasma with $v_{T x}=0.1 c, \omega=0.01 \omega_{p}, T_{x} / T_{z}=50$. Solid line shows the real part of the electric field profile obtained from the full solution making use of Eq.(10). Dashed line corresponds to the solution of Ref.[1] $E_{p 1} e^{i k_{p 1} z}$ given by Eq.(17). Dotted line corresponds to $E_{p 2} e^{i k_{p 2} z}$ given by Eq.(21). 


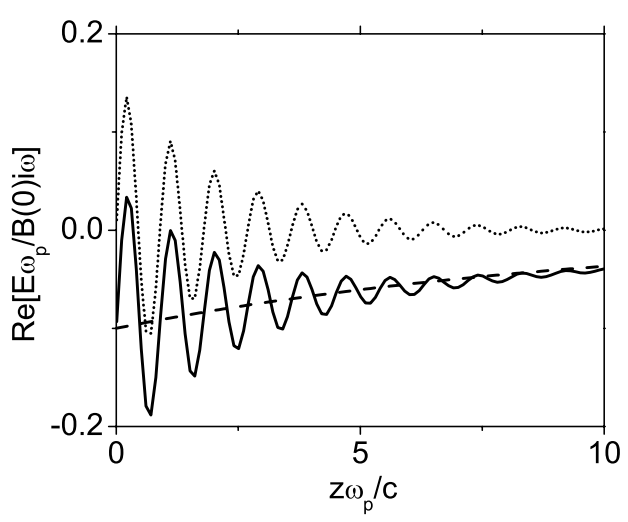

FIG. 1: 


\section{External Distribution}

Plasma Research Laboratory, Australian National University, Australia

Professor I.R. Jones, Flinders University, Australia

Professor João Canalle, Instituto de Fisica DEQ/IF - UERJ, Brazil

Mr. Gerson O. Ludwig, Instituto Nacional de Pesquisas, Brazil

Dr. P.H. Sakanaka, Instituto Fisica, Brazil

The Librarian, Culham Laboratory, England

Mrs. S.A. Hutchinson, JET Library, England

Professor M.N. Bussac, Ecole Polytechnique, France

Librarian, Max-Planck-Institut für Plasmaphysik, Germany

Jolan Moldvai, Reports Library, Hungarian Academy of Sciences, Central Research Institute for Physics, Hungary

Dr. P. Kaw, Institute for Plasma Research, India

Ms. P.J. Pathak, Librarian, Institute for Plasma Research, India

Ms. Clelia De Palo, Associazione EURATOM-ENEA, Italy

Dr. G. Grosso, Instituto di Fisica del Plasma, Italy

Librarian, Naka Fusion Research Establishment, JAERI, Japan

Library, Laboratory for Complex Energy Processes, Institute for Advanced Study, Kyoto University, Japan

Research Information Center, National Institute for Fusion Science, Japan

Dr. O. Mitarai, Kyushu Tokai University, Japan

Dr. Jiangang Li, Institute of Plasma Physics, Chinese Academy of Sciences, People's Republic of China

Professor Yuping Huo, School of Physical Science and Technology, People's Republic of China

Library, Academia Sinica, Institute of Plasma Physics, People's Republic of China

Librarian, Institute of Physics, Chinese Academy of Sciences, People's Republic of China

Dr. S. Mirnov, TRINITI, Troitsk, Russian Federation, Russia

Dr. V.S. Strelkov, Kurchatov Institute, Russian Federation, Russia

Professor Peter Lukac, Katedra Fyziky Plazmy MFF UK, Mlynska dolina F-2, Komenskeho Univerzita, SK-842 15 Bratislava, Slovakia

Dr. G.S. Lee, Korea Basic Science Institute, South Korea

Institute for Plasma Research, University of Maryland, USA

Librarian, Fusion Energy Division, Oak Ridge National Laboratory, USA

Librarian, Institute of Fusion Studies, University of Texas, USA

Librarian, Magnetic Fusion Program, Lawrence Livermore National Laboratory, USA

Library, General Atomics, USA

Plasma Physics Group, Fusion Energy Research Program, University of California at San Diego, USA

Plasma Physics Library, Columbia University, USA

Alkesh Punjabi, Center for Fusion Research and Training, Hampton University, USA

Dr. W.M. Stacey, Fusion Research Center, Georgia Institute of Technology, USA

Dr. John Willis, U.S. Department of Energy, Office of Fusion Energy Sciences, USA

Mr. Paul H. Wright, Indianapolis, Indiana, USA 
The Princeton Plasma Physics Laboratory is operated by Princeton University under contract with the U.S. Department of Energy.

\author{
Information Services \\ Princeton Plasma Physics Laboratory \\ P.O. Box 451 \\ Princeton, NJ 08543
}

Phone: 609-243-2750

Fax: 609-243-2751

e-mail: pppl_info@pppl.gov

Internet Address: http://www.pppl.gov 